\title{
Spectrally-Efficient WDM Nyquist Pulse-Shaped 16-QAM Subcarrier Modulation Transmission with Direct Detection
}

\author{
M. Sezer Erkılınç, Student Member, IEEE, Zhe Li, Student Member, IEEE, Stephan Pachnicke, Senior \\ Member, IEEE, Helmut Griesser, Member, IEEE, Benn C. Thomsen, Member, IEEE, Polina Bayvel, Fellow, IEEE, \\ and Robert I. Killey, Member, IEEE
}

\begin{abstract}
The ability to transmit signals with high information spectral density (ISD) using low complexity and costeffective transceivers is essential for short- and medium-haul optical communication systems. Consequently, spectrally-efficient direct detection transceiver-based solutions are attractive for such applications. In this paper, we experimentally demonstrate the wavelength division multiplexed (WDM) transmission of $7 \times 12$ GHz-spaced dispersion pre-compensated Nyquist pulseshaped 16-QAM subcarrier modulated channels operating at a net bit rate of $24 \mathrm{~Gb} / \mathrm{s}$ per channel, and achieving a net optical ISD of $2.0 \mathrm{~b} / \mathrm{s} / \mathrm{Hz}$. The direct detection receiver used in our experiment consisted of a single-ended photodiode and a single analog-to-digital converter (ADC). The carrier-to-signal power ratio (CSPR) at different values of optical signal-to-noise ratio (OSNR) was optimized to maximize the receiver sensitivity performance. The transmission experiments were carried out using a recirculating fiber loop with uncompensated standard single-mode fiber (SSMF) and EDFA-only amplification. The maximum achieved transmission distances for single channel and WDM signals were $727 \mathrm{~km}$ and $323 \mathrm{~km}$ below the bit-error ratio (BER) of $3.8 \times 10^{-3}$, respectively. To the best of our knowledge, this is the highest achieved ISD for WDM transmission in direct detection links over such distances.
\end{abstract}

Index Terms-Optical fiber communication, wavelength division multiplexing, information spectral density, direct detection, transceiver design, subcarrier modulation, Nyquist pulse shaping, single sideband signaling, digital signal processing.

\section{INTRODUCTION}

C ontinuously increasing high bandwidth demand due to data intensive services such as IP-TV, high-definition video-on-demand and cloud computing requires spectrallyefficient modulation formats in access, metropolitan and regional links [1], [2]. Multi-level and multi-dimensional modulation formats, e.g., quadrature amplitude modulation (QAM) with polarization multiplexing, using coherent receivers combined with digital signal processing (DSP)-based compensation of fiber impairments [3] enable the highest channel

Manuscript received February 13, 2015. This work was supported by the EU ERA-NET+ project PIANO+ IMPACT, EPSRC UNLOC EP/J017582/1 and FP7 project ASTRON.

M. Sezer Erkılınç, Zhe Li, Benn C. Thomsen, Polina Bayvel and Robert I. Killey are with the Optical Networks Group, Department of Electronic and Electrical Engineering at University College London, London WC1E 7JE, U.K. (e-mail: m.erkilinc@ee.ucl.ac.uk; zhe.li@ee.ucl.ac.uk; b.thomsen@ucl.ac.uk; p.bayvel@ucl.ac.uk; r.killey@ucl.ac.uk).

S. Pachnicke is with the ADVA Optical Networking SE, Maerzenquelle 1-3, 98617 Meiningen, Germany. (spachnicke@advaoptical.com).

H. Griesser is with the ADVA Optical Networking SE, Fraunhoferstr. 9a, 82152 Martinsried, Germany. (hgriesser@advaoptical.com). bit rates and information spectral densities (ISDs) [4], [5]. However, cost-effectiveness is another essential requirement for short- and medium-haul links $(\leq 800 \mathrm{~km})$ in which direct detection might be favorable due to its lower optical complexity. Thus, DSP-enabled transceivers employing direct detection (DD) receivers, i.e., consisting of only a singleended photodiode and a single analogue-to-digital converter (ADC), offer promising and practical solutions for metro and access links. Recently, service providers have started deploying $100 \mathrm{~Gb} / \mathrm{s}$ metro solutions based on $4 \times 28 \mathrm{~Gb} / \mathrm{s}$ DD technology [6].

In this paper, we describe experimental demonstrations of single channel and WDM transmission (12 GHz-spaced 7 channels) of single sideband (SSB) Nyquist pulse-shaped 16QAM subcarrier modulated signals using direct detection. The receiver consisted of only a single-ended photodiode and a single ADC. Back-to-back and transmission performance was assessed at a net bit rate of $24 \mathrm{~Gb} / \mathrm{s}$ per channel with a net optical ISD of $2.0 \mathrm{~b} / \mathrm{s} / \mathrm{Hz}$ (a gross bit rate of $25 \mathrm{~Gb} / \mathrm{s}$ with a gross optical ISD of $2.08 \mathrm{~b} / \mathrm{s} / \mathrm{Hz}$ including the harddecision forward error correction (HD-FEC) overhead). The variation of the optimum carrier-to-signal power ratio (CSPR) for different optical signal-to-noise ratio (OSNR) values was also investigated in simulations and experimentally verified. The accumulated dispersion along the fiber link was compensated using the electronic pre-distortion (EPD) technique, precompensation of the chromatic dispersion using transmitterbased DSP. The single channel and WDM signals were successfully transmitted over uncompensated standard singlemode fiber (SSMF) up to $727 \mathrm{~km}$ and $323 \mathrm{~km}$ below the biterror ratio (BER) of $3.8 \times 10^{-3}$, respectively.

\section{Review of the WDM DiRect Detection EXPERIMENTS (ISD OF $\geq 0.4 \mathrm{~B} / \mathrm{s} / \mathrm{HZ}$ )}

Numerous experimental demonstrations of spectrally-efficient direct detection WDM systems have been reported to date, utilizing a variety of formats, including pulse amplitude modulation (PAM), (optical) duobinary ((O)DB), (e.g., phase-shaped binary transmission (PSBT) [7]), subcarrier modulation (SCM), namely Nyquist-subcarrier modulation (Nyquist-SCM) and orthogonal frequency division multiplexing (OFDM), and carrierless amplitude/phase (CAP) modulation. The published experimental measurements of WDM 


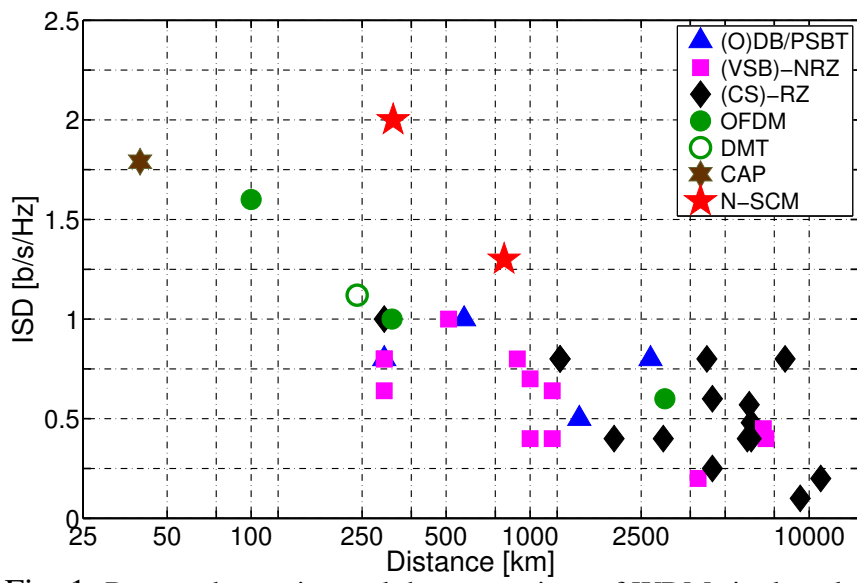

Fig. 1: Reported experimental demonstrations of WDM single polarization direct detection systems in terms of achieved net optical ISD versus distance. Formats: (VSB)-NRZ [8]-[15], (CS)-RZ [17]-[20], (O)DB [22]-[25], OFDM [29], [30], DMT [31] and Nyquist(N)-SCM [43] and this paper.

single polarization transmission performance for the different modulation formats are summarised in Fig. 1 in which the net ISD versus maximum distance is plotted.

On-off keying (OOK), also referred to as 2-PAM, is the simplest modulation format to generate and detect. Several non-return-to-zero (NRZ) OOK experiments at an ISD of $<0.5$ $\mathrm{b} / \mathrm{s} / \mathrm{Hz}$ without the use of optical filtering at the transmitter have been demonstrated for submarine links [8], [9], as shown in Fig. 1. The ISD can be increased to $1 \mathrm{~b} / \mathrm{s} / \mathrm{Hz}$ with optical sideband or narrow filtering. Vestigial sideband (VSB) OOK experiments have been reported with ISDs of from 0.64 to 1 b/s/Hz [10]-[15]. Moreover, return-to-zero (RZ) OOK pulses with duty cycles of $33 \%, 50 \%$, and $67 \%$ (carrier-suppressed RZ (CS-RZ)) can be generated using a pulse carver, typically a Mach-Zehnder modulator (MZM) driven sinusoidally [16]. Among the binary signaling formats, CS-RZ has the highest resilience to fiber nonlinearity. It has been successfully demonstrated with ISDs of between 0.4 and $0.8 \mathrm{~b} / \mathrm{s} / \mathrm{Hz}$ for submarine and transoceanic links [17]-[19] and $1 \mathrm{~b} / \mathrm{s} / \mathrm{Hz}$ for metro distances [20]. Moreover, duobinary signaling, implemented using either optical or electrical filtering after differential pre-coding [21], has been proposed for both long- [22] and medium-haul optical communication systems with ISDs of up to $1 \mathrm{~b} / \mathrm{s} / \mathrm{Hz}$ [23]-[25].

The ISD of all the aforementioned modulation techniques is limited to $1 \mathrm{~b} / \mathrm{s} / \mathrm{Hz}$ due to their binary coding. Nyquist pulse-shaped four-level pulse amplitude modulation (Nyquist 4-PAM) is a simple and low complexity multi-level format that potentially offers an ISD greater than $1 \mathrm{~b} / \mathrm{s} / \mathrm{Hz}$. However, it suffers from low receiver sensitivity as it uses only one degree-of-freedom. Yet, it is attractive for very short distances, e.g., interconnects and intra-datacenters networks [26]. Alternatively, DSP-based transceiver architectures, enabled by high-speed digital-to-analog and analog-to-digital converters (DACs/ADCs), offering ISDs greater than $1 \mathrm{~b} / \mathrm{s} / \mathrm{Hz}$ using QAM signaling have started to be investigated since they offer higher receiver sensitivity than 4-PAM. In such transceiver architectures, subcarrier modulation (SCM) (e.g., NyquistSCM and OFDM) can be used for QAM signaling since the amplitude and phase of each subcarrier, also referred to as RF-subcarrier(s), can be recovered after photodetection from the optical carrier-signal beating products.

Direct detection optical OFDM (DDO-OFDM) has attracted much research interest for access, metro and regional applications. It uses multiple orthogonal subcarriers for encoding the data stream [27] (in contrast to Nyquist-SCM which uses a single subcarrier). DDO-OFDM offers a promising solution for metro and access applications due to its resilience to chromatic dispersion [27], and the possibility to use adaptive modulation on each subcarrier to handle the frequency response of a nonideal link [28]. Recently, WDM DDO-OFDM experiments have been demonstrated with ISDs of $0.6 \mathrm{~b} / \mathrm{s} / \mathrm{Hz}$ over 3040 $\mathrm{km}$ [29], and $1.6 \mathrm{~b} / \mathrm{s} / \mathrm{Hz}$ over $100 \mathrm{~km}$ [30], as shown in Fig. 1. A real-valued OFDM signal, so-called discrete multi-tone (DMT), has also been demonstrated over $240 \mathrm{~km}$ of SSMF utilizing VSB signaling with an ISD of $1.12 \mathrm{~b} / \mathrm{s} / \mathrm{Hz}$ [31].

One disadvantage of SCM formats, such as DDO-OFDM and single subcarrier modulation, is that they suffer from signal-signal beating interference (SSBI). Although a spectral guard-band can be used between the optical carrier and sideband, this reduces the ISD. Although some proposed optical or DSP-based methods to mitigate/cancel the SSBI, allowing the width of the guard-band to be reduced, have been shown to be effective, they come at the price of either a significant degradation in OSNR performance [32], increased optical complexity [33], digital complexity [34] or overheads [35]. High peak-to-average power ratio (PAPR) due to the constructive interference of the subcarriers, leading to high peaks in signal waveform, is another drawback for OFDM signals. This leads to the requirement for using high optical carrier power, to maintain unipolar modulation, and consequently, high required OSNR. It also necessitates an increase in the required dynamic range of the DACs/ADCs. Clipping is a practical low complexity solution to reduce the PAPR of the OFDM driving signals [36]. However, it comes at the expense of nonlinear distortion and penalties [27], [37], [38].

As an alternative to DDO-OFDM, the QAM symbols can be transmitted using a single subcarrier, a technique termed single SCM, to avoid the issue of high PAPR. To obtain the highest possible ISD, Nyquist pulse shaping with sideband filtering can be used. It has been shown that Nyquist pulse-shaped SCM signal formats have lower PAPR compared to DDO-OFDM and higher tolerance to SSBI [39]. For this reason, we have investigated the Nyquist-SCM modulation technique to extend the transmission distances and information spectral densities of direct detection WDM systems. The next section describes the Nyquist pulse-shaped SCM system design including the transceiver architecture and the simulation model.

\section{NyQUisT PULSE-ShAPED SCM SYSTEM DESCRIPTION AND TRANSCEIVER DSP DESIGN}

In this section, first, the numerical simulations, that are carried out to optimize the Nyquist pulse-shaped SCM system characteristics to achieve a trade-off between the ISD and the required OSNR, are described. Then, the transceiver DSP design used in the simulations and experiments for offline signal generation and detection are outlined. The direct detection 


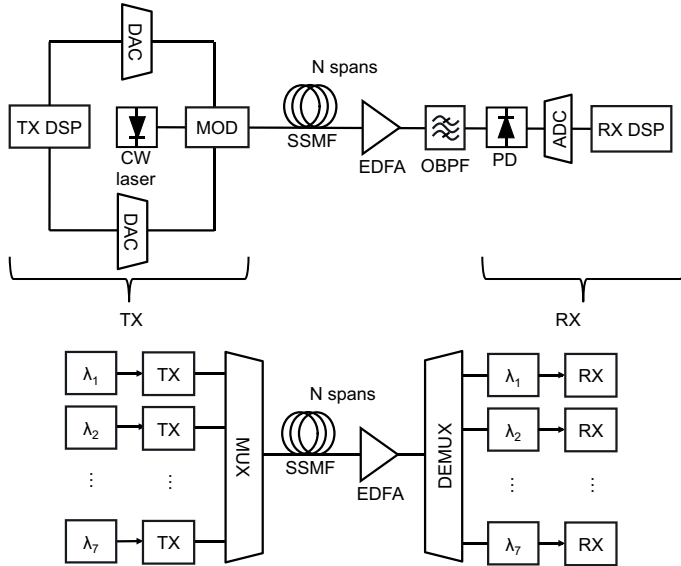

Fig. 2: System architecture for the single channel (top) and WDM system (bottom).

system architecture considered in this paper is shown in Fig. 2. The direct-detection SCM technique utilizes linear optical field modulation to quadrature amplitude modulate a single subcarrier with $M$ levels ( $M$-QAM) [40]. After transmission over a fiber link, an optical band-pass filter (OBPF) is used to demultiplex the channel of interest. Detection is performed using a single-ended photodiode. Finally, the detected signal is sampled and quantized using a single ADC.

The optical ISD is determined by the frequency spacing $\left(f_{s c}\right)$ between the optical carrier and the subcarrier, and the choice of double sideband (DSB) or single sideband (SSB) signaling, the latter offering the lowest possible channel spacing. For the case of the DSB SCM format with $f_{s c}$ equal to the symbol rate $\left(f_{s}\right)$, the maximum ISD approaches $\log _{2}(M) / 4$, referred to as single-cycle SCM [40]. The ISD can be increased towards $\log _{2}(M) / 2$ through the use of Nyquist pulse shaping, and a reduction in the subcarrier frequency from $f_{s}$ to $f_{s} / 2$, referred to as half-cycle Nyquist-SCM [41]. A description of Nyquist pulse shaping in optical communication systems can be found in [42]. Combining this approach with SSB signaling results in ISDs approaching $\log _{2}(M)$. The half-cycle Nyquist-SCM with a roll-off factor $(\alpha)$ of 0 was first experimentally demonstrated for single channel case in [41]. Although using Nyquist pulse shaping filters with such a low roll-off factor maximises the ISD, it comes at the expense of lower tolerance to SSBI and high PAPR with the corresponding drawbacks, as briefly described in the previous section and discussed extensively in [39]. Therefore, a non-zero value for $\alpha$ can be used to achieve a trade-off between the spectral efficiency and the required OSNR. For the experiments and simulations described below, the values of $\alpha$ and $f_{s c}$ were set to 0.3 and $0.75 \times f_{s}$, respectively, since they provide a good compromise point as investigated in [43]. The SSB Nyquist-SCM transmitter DSP design is shown in Fig. 3. We have previously demonstrated the single channel and WDM systems utilizing SSB Nyquist pulse-shaped QPSK SCM in back-to-back operation [44] and transmission over $800 \mathrm{~km}$ of SSMF [43] with a net optical ISD of $1.2 \mathrm{~b} / \mathrm{s} / \mathrm{Hz}$, plotted in Fig. 1.

Transmission simulations were carried out with MATLAB using the split-step Fourier method to solve the nonlinear Schrödinger equation (NLSE). Four $2^{18}$ patterns, based on a de Bruijn binary sequence and decorrelated by 0.25 of the

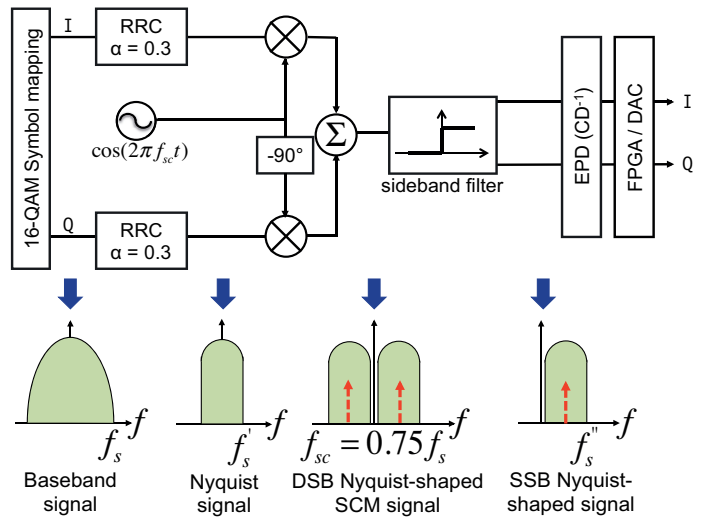

Fig. 3: Block diagram of Nyquist-SCM Transmitter DSP (top) and the schematic of the signal spectra of DSB and SSB three quarter-cycle $\left(f_{s c}=0.75 \times f_{s}\right)$ Nyquist pulse-shaped $(\alpha=0.3) \mathrm{SCM}$ (bottom). $f_{s}$ : symbol rate, $f_{s c}$ : subcarrier frequency, $f_{s}^{\prime}=f_{s} / 2(1+\alpha)$, $f_{s}^{\prime \prime}=f_{s c}+f_{s}^{\prime}$, EPD: Electronic pre-distortion and CD: Chromatic dispersion.

pattern length, were mapped to 16-QAM symbols. A pair of root raised-cosine (RRC) pulse-shaping filters with $\alpha=0.3$, 256 taps and a stop-band attenuation of $40 \mathrm{~dB}$ were applied to the in-phase (I) and quadrature $(\mathrm{Q})$ components to generate the digital representation of the Nyquist pulse-shaped signal. The I- and Q-components were up-converted to a subcarrier frequency $\left(f_{s c}\right)$ of $4.68 \mathrm{GHz}\left(0.75 \times f_{s}\right)$ and added to each other to obtain a DSB Nyquist pulse-shaped SCM signal at a bit rate of $25 \mathrm{~Gb} / \mathrm{s}$, as shown in Fig. 3. DSB to SSB conversion was performed using a digital sideband filter, followed by EPD, implemented in the frequency domain to mitigate the chromatic dispersion at the targeted distances. As described in [45], [46], the spectrum of the pre-dispersed signal, $E(\omega, 0)$ can be written as:

$$
\begin{aligned}
E(\omega, 0) & =E(\omega, L) H^{-1}(\omega) \\
& =E(\omega, L) \exp \left(j \frac{D}{4 \pi c} \lambda^{2} \omega^{2} L\right)
\end{aligned}
$$

where $E(\omega, L)$ is the desired optical field of the signal at the end of the link of length $(L), H^{-1}(\omega)$ is the inverse of the transfer function of the fiber (neglecting loss and nonlinearity), $\lambda$ is the wavelength, $c$ is the speed of light in vacuum, $D$ is the fiber dispersion and $\omega$ is the angular frequency. Finally, the real and imaginary parts of the pre-dispersed signal $\left(\operatorname{Re}\left\{F^{-1}[E(\omega, 0)]\right\}\right.$ and $\left.\operatorname{Im}\left\{F^{-1}[E(\omega, 0)]\right\}\right)$ were quantized to 6 bits (the nominal resolution of the DACs (Micram VEGA DACII) used in the experiments) and uploaded to the FPGARAM memory blocks. To demonstrate the fundamental limits of the proposed transceiver, simulations were performed assuming near ideal conditions, considering an effective number of bits (ENOB) of 6 bits and a sampling rate of $28 \mathrm{GSa} / \mathrm{s}$ while neglecting bandwidth limitations. Then, the practical parameters were taken into account, i.e., setting the electrical signalto-noise (SNR) to $23 \mathrm{~dB}$ to take into account the ENOB of the practical DACs (3.8 bits at $10 \mathrm{GHz}$ ) and, applying electrical $5^{\text {th }}$-order Bessel low-pass filters (LPFs) at the transmitter and receiver sides with bandwidths of 7 and $16 \mathrm{GHz}$, respectively to emulate the frequency response of the anti-imaging/-aliasing filters. To model the WDM transmission, $7 \times 12 \mathrm{GHz}$-spaced 
channels carrying $25 \mathrm{~Gb} / \mathrm{s}$ SSB Nyquist pulse-shaped 16-QAM SCM signals were generated and decorrelated by 490 samples.

The transmission link considered in the simulations consisted of uncompensated SSMF and EDFAs with a noise figure of $5 \mathrm{~dB}$. The fiber parameters $\alpha, D, \gamma$ and $L_{\text {span }}$ were chosen as $0.2 \mathrm{~dB} / \mathrm{km}, 17 \mathrm{ps} /(\mathrm{nm} . \mathrm{km}), 1.2 \mathrm{~W}^{-1} \mathrm{~km}^{-1}$ and $80 \mathrm{~km}$, respectively. An additional loss of $15 \mathrm{~dB}$ per amplifier span was included to take into account the effects of additional optical components. All amplified spontaneous emission (ASE)-noise generated by the EDFAs was added inline to model nonlinear signal-ASE noise interaction. The symmetric split-step Fourier method [47] was utilized to model the single channel and WDM transmission at a simulation bandwidth of $200 \mathrm{GHz}$ with step sizes of $1 \mathrm{~km}$ and $400 \mathrm{~m}$, respectively.

To demultiplex the channel of interest and remove the out-of-band ASE-noise before the photodiode, first an ideal brickwall-shaped OBPF for ideal system simulations and subsequently, a $4^{\text {th }}$-order super-Gaussian OBPF for the practical system simulations were applied. The transmitted optical signal was detected by a single-ended photodiode with a responsivity of $1 \mathrm{~A} / \mathrm{W}$, followed by an ADC with a resolution of 5bits and a sampling rate of $50 \mathrm{GSa} / \mathrm{s}$, and finally the receiver DSP, a block diagram of which is shown in Fig. 4. After the digitized received signal was normalized and resampled to 2 samples per symbol, the signal was split into two branches and down-converted to generate baseband I- and Q-components. Then, a pair of matched RRC filters with $\alpha=0.3$ were applied to the components, separately. Before the symbol-to-bit demapping, 5-tap FIR filtering was performed for symbol retiming. The cost function was chosen as the constant modulus algorithm to update the filter taps for fast convergence, before switching to decision directed least-mean squares (LMS). Finally, the bit-error ratio (BER) was calculated over $2^{20}$ bits by error counting. The transmission results are presented in terms of $Q^{2}$-factor $\left(Q^{2}[\mathrm{~dB}]=20 \log _{10}\left(\sqrt{2} \operatorname{erfcinv}\left(2 p_{b}\right)\right)\right.$ where $p_{b}$ is the BER. To calculate the net bit rate and net ISD, the hard decision decoding bound for the binary symmetric channel was utilized such that the maximum code rate $(r)$ is given by [48]

$$
r=1+p_{b} \log _{2} p_{b}+\left(1-p_{b}\right) \log _{2}\left(1-p_{b}\right) .
$$

At a BER of $3.8 \times 10^{-3}, r$ was found to be 0.96 . Hence, the net bit rate per channel was $24 \mathrm{~Gb} / \mathrm{s}(25 \mathrm{~Gb} / \mathrm{s} \times r)$, resulting in a net optical ISD of $2.0 \mathrm{~b} / \mathrm{s} / \mathrm{Hz}(2.08 \mathrm{~b} / \mathrm{s} / \mathrm{Hz} \times r)$ for the WDM transmission. The simulation results for back-toback and transmission cases are discussed together with the experimental results in Section V.

\section{TRANSMISSION EXPERIMENTS}

The optical transmission test-bed consisted of a $7 \times 25 \mathrm{~Gb} / \mathrm{s}$ channel SSB Nyquist pulse-shaped 16-QAM SCM transmitter, an optical fiber recirculating loop and a direct detection receiver to detect the channel of interest, as shown in Fig. 5. An external cavity laser (ECL) with a linewidth of $100 \mathrm{kHz}$ at $1550 \mathrm{~nm}$ was used as the seed for an optical comb generator (OCG) based on cascaded amplitude and phase modulators to generate seven $12 \mathrm{GHz}$-spaced unmodulated optical channels, as shown in Fig. 5. Although a laser source with higher

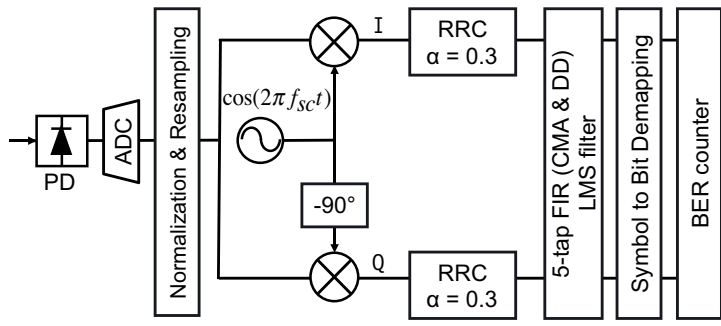

Fig. 4: Block diagram of Nyquist-SCM Receiver DSP. CMA: Constant modulus algorithm, DD: Decision-directed, LMS: Least mean squares and FIR: Finite-impulse response.

linewidth could be used in direct detection SCM, an ECL was used due to its availability. The number of comb lines was limited to seven in order to maintain the power variation across the channels to within $1 \mathrm{~dB}$. The channel spacing was chosen to ensure the penalty caused by linear crosstalk due to the neighboring channels was $\leq 1 \mathrm{~dB}$. Odd $\left(\lambda_{1,3,5,7}\right)$ and even $\left(\lambda_{2,4,6}\right)$ channels were separated using three cascaded Kylia micro-interferometer interleavers with a suppression of $40 \mathrm{~dB}$ to allow independent modulation with uncorrelated bit sequences.

As shown in Fig. 3(a), the signal waveforms used for driving the IQ-modulators were generated offline in MATLAB using four $2^{15}$ de Bruijn bit sequences, de-correlated by 0.25 of the pattern length. The waveforms were uploaded to the memory of a pair of Xilinx Virtex-5 FPGAs to drive the DACs. To prevent crosstalk between WDM channels due to the images generated by the DACs, electrical anti-imaging filters, $5^{\text {th }}$ order Bessel LPFs with a bandwidth of $7 \mathrm{GHz}$, were used. An optical SSB signal with an OSNR of $34 \mathrm{~dB}$ at a resolution of $0.1 \mathrm{~nm}$ was generated using the IQ-modulators with a switching voltage $\left(V_{\pi}\right)$ of $3.5 \mathrm{~V}$. The modulators were driven by the electrical signals with a peak-to-peak voltage $\left(V_{p p}\right)$ of $3.4 \mathrm{~V}$. Note that the IQ-modulators were biased close to their quadrature points to achieve approximately linear mapping from the electrical to the optical domain with the bias voltages, adjusted to achieve the desired optical carrier power. In direct detection links, the optimum optical carrier power value is dependent on the sideband power and is specified as the carrier-to-signal power ratio (CSPR):

$$
\operatorname{CSPR}(\mathrm{dB})=10 \log _{10}\left(\frac{P_{C}}{P_{S}}\right)
$$

where $P_{C}$ and $P_{S}$ are the optical carrier and sideband powers, respectively. The CSPR is discussed in more detail in Section V-A. Decorrelating fiber of $1 \mathrm{~km}$ length (delay of $17 \mathrm{~ns}$ corresponding to approximately 425 samples) was used to decorrelate the odd and even channels before they were combined and launched into the recirculating loop. The optical spectra of modulated odd and even channels are shown together in Fig. 5(b). Note that the optical spectra shown in Fig. 5(a)-(c) are taken from the optical spectrum analyser $(\mathrm{OSA})$ at a resolution of $0.01 \mathrm{~nm}$.

The transmission experiment was performed using an optical recirculating loop with a single span of $80.7 \mathrm{~km}$ $\left(D_{S S M F}=17 \mathrm{ps} / \mathrm{nm} / \mathrm{km}\right)$, as shown in Fig 5. The loop was controlled by two switches (AOM Tx and AOM Loop) to switch from 'signal $\mathrm{TX}^{\prime}$ ' to 'signal Loop' stage. An OBPF 


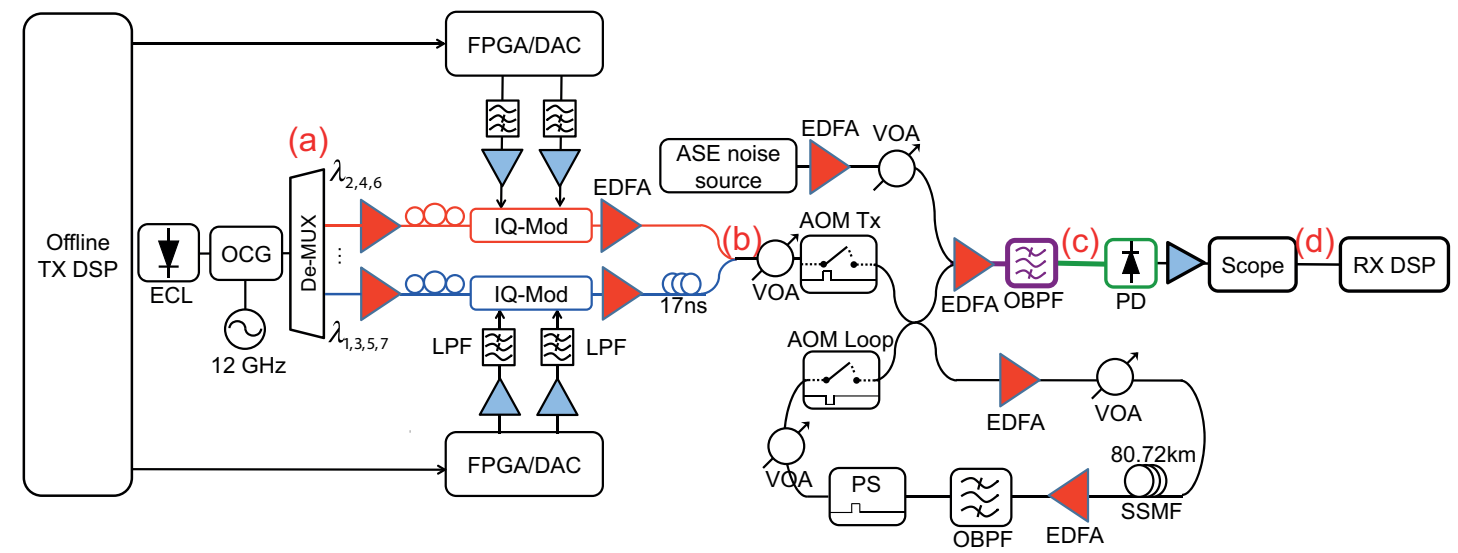

(a)

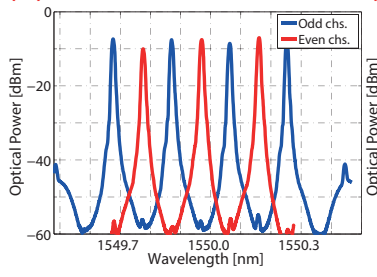

(b)

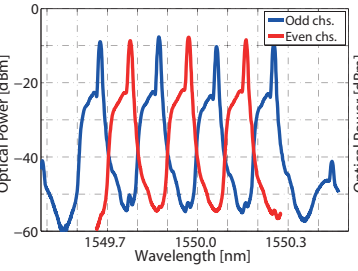

(c)

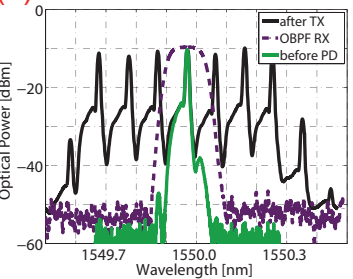

(d)

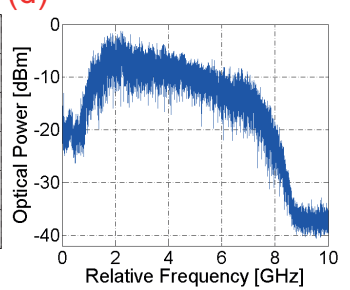

Fig. 5: Experimental setup for WDM SSB Nyquist pulse-shaped SCM transmission. Note that the block diagram of offline TX and RX DSP are presented in Fig. 3 and Fig. 4, respectively. FPGA: Field programmable gate array, VOA: Variable optical attenuator, AOM: Acoustooptic modulator, PS: Polarization scrambler, PD: Photodiode. Insets: Experimental optical spectra of 7 channels (a) after OCG, (b) after the transmitter and (c) transmitted optical signal, OBPF response at the receiver and received optical signal. (d) Received electrical spectrum.

(Yenista Optics XTM50-Wide) with a 3dB bandwidth of 200 $\mathrm{GHz}$ and a filter edge gradient of $500 \mathrm{~dB} / \mathrm{nm}$ was used to filter the out-of-band ASE-noise during the transmission. A loop synchronous polarization scrambler was utilized to randomize the signal polarization state in each span. The launch power into the span was controlled by the variable optical attenuators (VOAs). The fiber loss of $16 \mathrm{~dB}$ plus the combined loss of $15 \mathrm{~dB}$ from the VOAs, PS, AOM and OBPF resulted in a total loss per recirculation of $31 \mathrm{~dB}$, which was compensated by two EDFAs with a noise figure of $5 \mathrm{~dB}$, operating at their saturation point (18 $\mathrm{dBm}$ output power).

At the receiver, the channel of interest was demultiplexed using a tunable OBPF (Yenista Optics XTM50-Ultrafine) with a $3 \mathrm{~dB}$ bandwidth of $11 \mathrm{GHz}$ and a filter edge gradient of $800 \mathrm{~dB} / \mathrm{nm}$, as shown in Fig. 5(c). The filter was tuned manually to optimize the system performance. The demultiplexed optical signal was detected by a single-ended photodiode, followed by an RF-amplifier to boost the signal power. The amplified electrical signal was acquired using a single ADC (Tektronix DPO 72004 oscilloscope) operating at $50 \mathrm{GSa} / \mathrm{s}$ with an electrical bandwidth of $16 \mathrm{GHz}$ and a nominal resolution of 8 bits (ENOB of 5 bits at $10 \mathrm{GHz}$ ). The digitized signal spectrum is shown in Fig. 5(d). The signal was decoded for BER counting, as described in Section III with the block diagram illustrated in Fig. 4. The BER was calculated over $2^{20}$ bits by error counting and the $Q^{2}$-factor was computed from the measured BER.

\section{TRANSMISSION RESUlTS AND DISCUSSIONS}

The BER and $Q^{2}$-factor measurements were carried out for single channel and WDM systems operating at a bit rate 25 $\mathrm{Gb} / \mathrm{s}$. The signal quality was assessed first in back-to-back, and then after transmission over a variety of distances. ASE-noise loading to characterize back-to-back operation was performed at the receiver. To optimize the system performance, carrierto-signal power ratio (CSPR) values were varied at different OSNR values. For WDM transmission, the BER was measured for all seven transmitted channels.

\section{A. Back-to-back Performance}

Simulated and experimentally measured back-to-back BERs with respect to OSNR for single channel and WDM systems are presented in Fig. 6. Ideal system simulations, neglecting practical limitations such as DACs/ADC quantization noise and optical/electrical filtering effect, indicated that the required OSNR at the HD-FEC threshold in single channel back-toback operation is $21 \mathrm{~dB}$ for the SSB Nyquist pulse-shaped 16QAM SCM signal with $\alpha=0.3$ and $f_{s c}=4.68 \mathrm{GHz}\left(0.75 \times f_{s}\right)$. In our experiments, the required OSNR for single channel was measured to be $23 \mathrm{~dB}$, a $2 \mathrm{~dB}$ implementation penalty with respect to the ideal simulation results. This was accounted for by the DACs/ADC quantization noise, low-pass filtering and non-ideal optical filtering at the receiver. Moreover, for the WDM system, an additional $1 \mathrm{~dB}$ penalty was observed due to the linear crosstalk caused by the neighboring channels. This additional penalty was not observed in ideal simulations because a brickwall-shaped OBPF was used to demultiplex the channel of interest. The implementation penalties both in single channel and WDM systems were verified by simulations using practical parameters, as shown with blue and red solid lines in Fig. 6. To take into account the non-idealities of our experiment, the SNR of the electrical driving signals was set to $23 \mathrm{~dB}$, emulating the DACs' ENOB, and ADC resolution was set to 5 bits. In addition to quantization noise, a $4^{\text {th }}$-order super-Gaussian-shaped OBPF with a bandwidth of $11 \mathrm{GHz}$ was used to filter out the out-of-band ASE-noise 
and demultiplex the central WDM channel. There is good agreement between experimental and simulated BER values and constellations for single channel and WDM systems, as can be seen in Fig. 6.

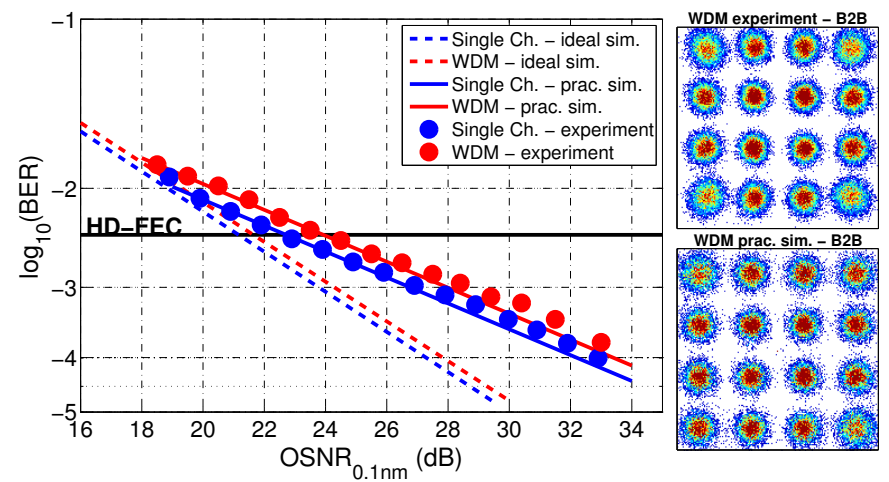

Fig. 6: Simulated and experimental BER vs OSNR curves for back-to-back case (left). Experimental and simulated WDM signal constellations at an OSNR of $34 \mathrm{~dB}$ (right).

In subcarrier modulation-based direct detection systems, it is crucial to optimize the CSPR to achieve the minimum BER at a given OSNR. Thus, we investigated the system sensitivity to CSPR variation, both in simulations and experimentally. To measure the CSPR value at a given OSNR accurately, two different methods were assessed. First, the transmitted signal was split into two arms and, one arm was detected using a coherent receiver whilst the other arm was detected by the direct detection receiver for BER counting, as explained in Section IV. After the optical full-field was recovered using the coherent receiver, two brickwall-shaped digital filters were used to filter out the optical carrier and sideband. The corresponding power values for the carrier and sideband were computed and the resulting CSPR value was determined using Eq.3. Since the measurement was carried out at a very high resolution (approximately $100 \mathrm{kHz}$ ), it is the most accurate method to measure the CSPR. In a simpler alternative approach, we measured the CSPR at a given OSNR using the corresponding optical spectrum taken from the OSA at a resolution of $0.01 \mathrm{~nm}$. After the spectrum was acquired, the carrier and sideband power were measured using two $5^{\text {th }}$ order super-Gaussian-shaped filters in MATLAB, similar to the first method. Finally, the measured CSPR values using the two different methods were compared and a discrepancy of approximately $10 \%$ was found. Since the second, simpler method was sufficiently accurate for our demonstrations, the majority of the CSPR values were measured using the second method.

The BER as a function of CSPR for 6 different OSNR values is shown in Fig. 7. The CSPR value was varied between 4 and $16 \mathrm{~dB}$ for OSNR values ranging from 19 to $29 \mathrm{~dB}$. The dashed red arrow indicates how the optimum CSPR shifts towards higher values as the OSNR is increased. At low CSPR values, the system is SSBI-limited since the signalsignal mixing products distort the desired signal (carrier-signal mixing products) severely. On the other hand, in the high CSPR regime, signal-ASE beating noise limits the system performance. The simulation results shown with the solid lines closely match the experimental results shown with the markers in Fig. 7. It was found that optimum CSPR values were similar for single channel and WDM systems.

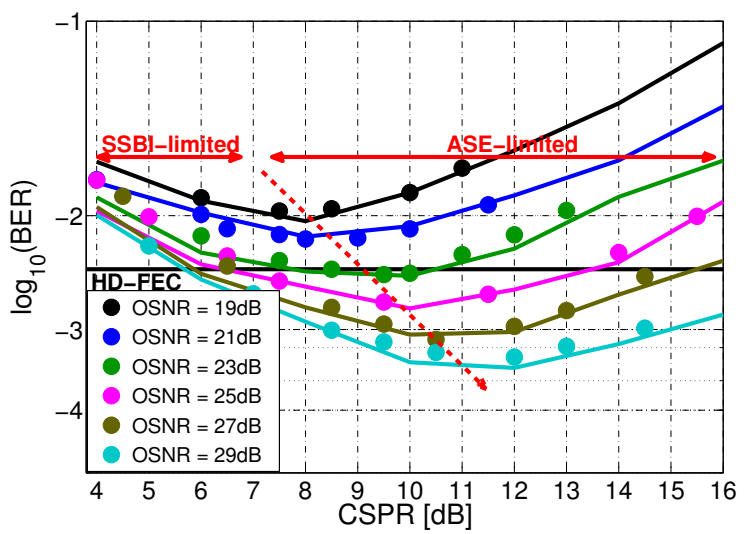

Fig. 7: Simulated and experimental BER with respect to CSPR at different OSNR levels in back-to-back operation. The dashed red arrow indicates the shift in the optimum CSPR value.

\section{B. Transmission Performance}

After back-to-back characterization, single channel and WDM transmission experiments were performed. Single channel transmission was carried out for distances from $242 \mathrm{~km}$ up to $727 \mathrm{~km}$ (up to 9 spans) using the recirculating fiber loop. The experimental $Q^{2}$-factor values for single channel transmission distances of up to $727 \mathrm{~km}$ and the simulated $Q^{2}$-factor values for $727 \mathrm{~km}$ transmission with respect to the launch power per channel are shown in Fig. 8. It was found that the simulated and experimental results are similar which can also be observed in the constellations shown in Fig. 8. In single channel transmission, the optimum launch power was found to be approximately $1 \mathrm{dBm}$. BER values below the FEC threshold were obtained for launch powers over the range from $-4.0 \mathrm{dBm}$ to $+4 \mathrm{dBm}$ at $242 \mathrm{~km}$. This range decreased when the transmission distance increased as expected. The maximum achieved transmission distance was $727 \mathrm{~km}$ at a launch power of $1 \mathrm{dBm}$ with the measured BER/ $Q^{2}$-factor of $3.5 \times 10^{-3} / 8.7 \mathrm{~dB}$, just achieving the HD-FEC threshold. The optimum CSPR value was found to be approximately $8 \mathrm{~dB}$ at $727 \mathrm{~km}$ in single channel transmission.

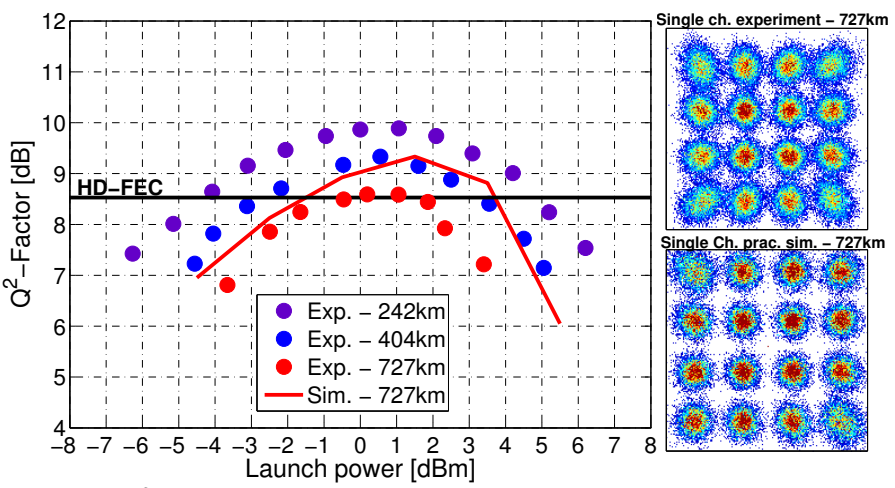

Fig. 8: $Q^{2}$-factor vs. launch power per channel for single channel (left) and corresponding experimental and simulated constellations at a distance of $727 \mathrm{~km}$ and at the optimum launch power of $1 \mathrm{dBm}$.

The 7-channel WDM transmission distances of 242 and $323 \mathrm{~km}$ were achieved with maximum $Q^{2}$-factors of 9 and 


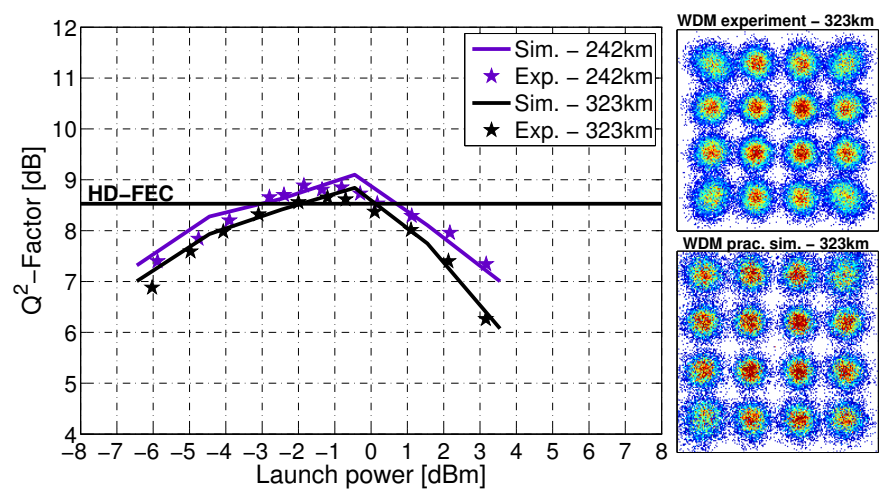

Fig. 9: $Q^{2}$-factor vs. launch power per channel for WDM system (left) and corresponding experimental and simulated constellations at a distance of $323 \mathrm{~km}$ and at the optimum launch power of $-1.6 \mathrm{dBm}$.

$8.7 \mathrm{~dB}$, respectively, at a launch power of $-1.6 \mathrm{dBm}$ per channel, as shown in Fig. 9 with their corresponding constellations. In the linear regime, WDM achieves the same $Q^{2}$ factor value at a slightly higher launch power per channel compared to the single channel transmission. This is due to the linear crosstalk caused by the neighbouring channels which was also observed in the back-to-back operation (see Fig. 6). Due to the additional inter-channel nonlinear effects during WDM transmission compared to single channel transmission, the maximum transmission distance was reduced from 727 to $323 \mathrm{~km}$. There is very good agreement between the transmission experiments and simulations. The optimum CSPR value was found to be approximately $7 \mathrm{~dB}$ in WDM transmission at $323 \mathrm{~km}$, slightly lower than the single channel transmission at $727 \mathrm{~km}$. This small change in the optimum CSPR value is due to the trade-off between the SSBI and fiber nonlinearities, and, as expected, the optimum CSPR value is lower in WDM transmission because of the fiber nonlinearities.

All 7 channels operating at $25 \mathrm{~Gb} / \mathrm{s}$ achieved a BER $<3.8 \times$ $10^{-3}\left(Q^{2}\right.$-factor $\left.>8.52 \mathrm{~dB}\right)$ at the optimum launch power of $1.6 \mathrm{dBm}$ per channel, as shown in Fig. 10. Hence, a total net bit rate of $168 \mathrm{~Gb} / \mathrm{s}(7 \mathrm{ch} . \times 25 \mathrm{~Gb} / \mathrm{s} \times r)$ with a net ISD of $2.0 \mathrm{~b} / \mathrm{s} / \mathrm{Hz}$ was achieved over $323 \mathrm{~km}$ of SSMF. This is plotted in Fig. 1 and, relative to the previous experiments, it is at the highest ISD reported for direct detection systems at this transmission distance.

It is of interest to compare the complexity of the optical hardware of the Nyquist-SCM system with that of an equivalent coherent polarization division multiplexed (PDM) 16QAM targeting the same bit rate. Assuming the same electrical bandwidth for both systems, and with the transmitter DSP approach described in this paper, four WDM channels are required to reach the same data rate as opposed to single PDM 16-QAM channel (e.g., $4 \times 28 \mathrm{~Gb} / \mathrm{s}$ at 7 GBaud with 16-QAM Nyquist-SCM using direct detection versus $1 \times 112$ $\mathrm{Gb} / \mathrm{s}$ at $14 \mathrm{GBaud}$ with coherent PDM 16-QAM). However, the single sideband Nyquist-SCM scheme can be also realized shifting the optical carrier frequency by the IQ-modulators during modulation, enabling the full bandwidth of the IQmodulators to be used as described in [34], [49]. Consequently, this configuration would allow a symbol rate of 14 GBaud using a single IQ-modulator, and hence, the required number of channels can be halved (e.g., $2 \times 56 \mathrm{~Gb} / \mathrm{s}$ at 14 GBaud).
In this case, the direct detection Nyquist-SCM system would require two transmitter lasers, two IQ-modulators and two photodiodes. In contrast, the coherent PDM 16-QAM system requires a single transmitter and a local oscillator laser, two IQ-modulators, and eight photodiodes (4 balanced detectors), in addition to polarization beam splitting/combining optics and a pair of optical hybrids. The simplified optical structure of the Nyquist-SCM scheme comes at the expense of lower maximum transmission distance and information spectral density since only a single polarization is used and optical filtering, rather than digital filtering, determines the achievable channel spacing.

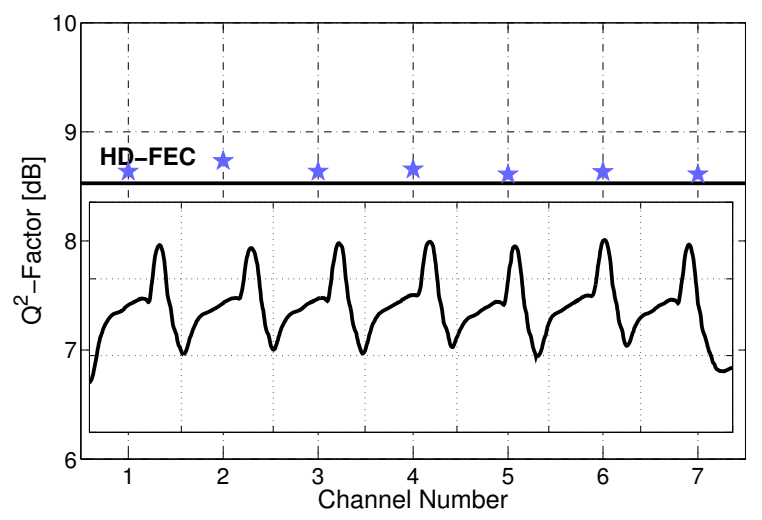

Fig. 10: $Q^{2}$-factor for each received channel at $323 \mathrm{~km}$. Inset: Transmitted optical spectrum.

\section{Summary AND CONCLUSIONS}

We experimentally demonstrated, for the first time, single channel and WDM Nyquist pulse-shaped 16-QAM subcarrier modulation with a net optical information spectral density of $2.0 \mathrm{bits} / \mathrm{s} / \mathrm{Hz}$ using a simple direct detection receiver (a single-ended photodiode and a single ADC). Back-to-back and transmission performance was assessed for ideal and practical configurations. First, simulations of the ideal system (with minimal DACs/ADC quantization noise and neglecting electrical bandwidth limitations) for the back-to-back case were performed to investigate the fundamental limits of the proposed system and to quantify the implementation penalties of the practical system $(2 \mathrm{~dB}$ for single channel and $3 \mathrm{~dB}$ for WDM). Then, the experimental results on single channel and 7-channel WDM system performance for back-to-back and transmission cases were verified by simulations of the practical system. The effect of varying the carrier-to-signal power ratio at different OSNR values was also simulated and experimentally measured for the back-to-back case.

The maximum achievable transmission distance over EDFAonly amplified SSMF with $31 \mathrm{~dB}$ loss per span (the fiber loss plus the total insertion loss due to the loop components) was $727 \mathrm{~km}$ for the single channel case, whereas the transmission distance decreased to $323 \mathrm{~km}$ in WDM case mainly due to inter-channel nonlinear effects. The electronic pre-distortion technique, pre-dispersing the signal at the transmitter DSP, was utilized to mitigate the chromatic dispersion accumulated along the fiber. To the best of our knowledge, this is the highest achieved ISD, at this distance, among the reported experimental WDM demonstrations in direct detection links 
using a single-ended photodiode and a single ADC. The results indicate that direct detection Nyquist pulse-shaped SCM can be an attractive approach offering high information spectral density and cost-effective transceiver design, and thus, may be practical for metro, regional and access applications.

\section{REFERENCES}

[1] Alcatel-Lucent, "Bell Labs metro network traffic growth: An architecture impact study," Strategic White Paper, Dec. 2013.

[2] Cisco, "Cisco visual networking index: Forecast and methodology, 2013$2018, " 2014$

[3] S. Savory, "Digital filters for coherent optical receivers," Opt. Express, vol. 16, no. 2, pp. 804-817, 2008.

[4] M. Mazurczyk, "Spectral shaping in long haul optical coherent systems with high spectral efficiency," J. Lightw. Technol., vol. 32, no. 16, pp. 2915-2924, 2014.

[5] J.X. Cai, "100G transmission over transoceanic distance with high spectral efficiency and large capacity," J. Lightw. Technol., vol. 30, no. 24, pp. 3845-3856, 2012.

[6] ADVA, Efficient 100G transport (2014). http://www.advaoptical.com/en/innovation/100g-transport.aspx (retrieved February $11^{\text {th }}$ 2015)

[7] D. Penninckx et al., "The phase-shaped binary transmission (PSBT): A new technique to transmit far beyond the chromatic dispersion limit," IEEE Photon. Technol. Lett., vol. 9, no. 2, pp. 259-261, 1997.

[8] C.R. Davidson et al., " $1800 \mathrm{~Gb} / \mathrm{s}$ transmission of one hundred and eighty $10 \mathrm{~Gb} / \mathrm{s}$ QDM channels over $7000 \mathrm{~km}$ using the full EDFA C-band," in Proc. $(O F C)$, Baltimore, MD, USA, 2000, post-deadline paper PD25.

[9] G. Vareille et al., "3 Tbit/s $(300 \times 11.6 \mathrm{Gbit} / \mathrm{s})$ transmission over 7380 $\mathrm{km}$ using $\mathrm{C}+\mathrm{L}$ band with $25 \mathrm{GHz}$ channel spacing and NRZ format," in Proc. OFC, Amsterdam, Netherlands, 2001, post-deadline paper PD22.

[10] S. Bigo et al., "Transmission of $125 \mathrm{WDM}$ channels at $42.7 \mathrm{Gbit} / \mathrm{s}$ (5 Tbit/s capacity) over $12 \times 100 \mathrm{~km}$ of TeraLight Ultra fibre," in Proc. ECOC, Amsterdam, Netherlands, 2001, post-deadline paper PD.M.1.1.

[11] W. Idler et al., "Vestigial side-band demultiplexing for ultra-high capacity $(0.64 \mathrm{bit} / \mathrm{s} / \mathrm{Hz})$ of $128 \times 40 \mathrm{Gbit} / \mathrm{s}$ channels," in Proc. OFC, Anaheim, CA, USA, 2001, paper MM3.

[12] S. Bigo et al., "5.12 Tbit/s $(128 \times 40 \mathrm{Gbit} / \mathrm{s}$ WDM $)$ transmission over $3 \times 100 \mathrm{~km}$ of TeraLight fiber," in Proc. ECOC, Munich, Germany, 2000, post-deadline paper PD1.2.

[13] W. Idler et al., " $0.8 \mathrm{bit} / \mathrm{s} / \mathrm{Hz}$ of information spectral density by vestigial

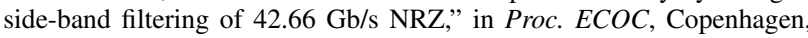
2002, paper 8.1.5.

[14] G. Charlet et al., "3.2 Tbit/s $(80 \times 42.7 \mathrm{~Gb} / \mathrm{s})$ C-band transmission over $9 \times 100 \mathrm{~km}$ of TeraLight fiber with $50 \mathrm{GHz}$ channel spacing," in Proc. Top. Meeting Opt. Amps. and Their Apps., Vancouver, Canada, 2002, post-deadline paper PDP1.

[15] K. Schuh et al., "8 Tbit/s $(80 \times 107 \mathrm{Gbit} / \mathrm{s})$ DWDM ASK-NRZ VSB transmission over $510 \mathrm{~km}$ NZDSF with $1 \mathrm{bit} / \mathrm{s} / \mathrm{Hz}$ spectral efficiency," in Proc. ECOC, Berlin, Germany, 2007, post-deadline paper PD1.8

[16] E. Ip and J.M. Kahn, "Power spectra of return-to-zero optical signals," J. Lightw. Technol., vol. 24, no. 3, pp. 1610-1618, 2006.

[17] J.-X. Cai et al., "Transmission of thirty-eight $40 \mathrm{~Gb} / \mathrm{s}$ channels $(>1.5$ $\mathrm{Tb} / \mathrm{s}$ ) over transoceanic distance," in Proc. OFC, Anaheim, CA, USA, 2002, post-deadline paper FC4.

[18] Y. Frignac et al., "Transmission of 256 wavelength-division and polarization-division multiplexed channels at $42.7 \mathrm{~Gb} / \mathrm{s}(10.2 \mathrm{~Tb} / \mathrm{s}$ capacity) over $3 \times 100 \mathrm{~km}$ of TeraLight fiber," in Proc.OFC., Anaheim, CA, USA, 2002, post-deadline paper FC5.

[19] D.F. Grosz et al., " $5.12 \mathrm{~Tb} / \mathrm{s}(128 \times 42.7 \mathrm{~Gb} / \mathrm{s})$ transmission with 0.8 $\mathrm{bit} / \mathrm{s} / \mathrm{Hz}$ spectral efficiency over $1280 \mathrm{~km}$ of standard single-mode fiber using all-Raman amplification and strong signal filtering," in Proc. ECOC, Copenhagen, 2002, post-deadline paper PD4.3.1.

[20] I. Morita et al., " $100 \%$ Spectral-efficient $25 \times 42.7 \mathrm{Gbit} / \mathrm{s}$ transmission using asymmetric filtered CS-RZ signal and a novel crosstalk suppressor," in Proc. ECOC, Copenhagen, 2002, post-deadline paper PD4.7.

[21] E. Pincemin et al., "Experimental performance comparison of duobinary and PSBT modulation formats for long-haul $40 \mathrm{~Gb} / \mathrm{s}$ transmission on G 0.652 fibre," Opt. Express, vol. 20, no. 27, pp. 28171-28190, 2012.

[22] G. Charlet et al., "Cost-optimized $6.3 \mathrm{Tbit} / \mathrm{s}$-capacity terrestrial link over $17 \times 100 \mathrm{~km}$ using phase-shaped binary transmission in a conventional all-EDFA SMF-based system, in Proc. OFC, Atlanta, Georgia, USA, 2003, post-deadline paper PD2.5.
[23] T. Ono et al., "Characteristics of optical duobinary signals in terabit/s capacity, high-spectral efficiency WDM systems," J. Lightw. Technol., vol. 16, no. 5, pp. 788-797, 1998.

[24] H. Bissessur et al., "3.2 Tbit/s $(80 \times 40 \mathrm{Gbit} / \mathrm{s})$ phase-shaped binary transmission over $3 \times 100 \mathrm{~km}$ with $0.8 \mathrm{bit} / \mathrm{s} / \mathrm{Hz}$ efficiency," Electron. Lett., vol. 38, no. 8, pp. 377-379, 2002.

[25] M. Alfiad and S. Tibuleac, "100G superchannel transmission using $4 \times 28 \mathrm{~Gb} / \mathrm{s}$ subcarriers on a $25-\mathrm{GHz}$ Grid," IEEE Photon. Technol. Lett., vol. 27, no. 2, pp. 157-160, 2015

[26] J.L. Wei et al., "Performance and power dissipation comparisons between $28 \mathrm{~Gb} / \mathrm{s}$ NRZ, PAM, CAP and optical OFDM systems for data communication applications", J. Lightw. Technol., vol. 30, no. 20, pp. 3273-3280, 2012.

[27] A.J. Lowery and L.B. Du, "Optical orthogonal division multiplexing for long haul optical communications: A review of the first five years," Opt. Fiber Technol., vol. 17, pp. 421-438, 2011, invited paper.

[28] E. Giacoumidis et al., "Adaptive loading algorithms for IMDD optical OFDM PON systems using directly modulated lasers," J. Opt. Commun. and Networking, vol. 4, no. 10, pp. 769-778, 2012.

[29] Z. Li et al., "432-Gb/s direct-detection optical OFDM superchannel transmission over 3040-km SSMF," IEEE Photon. Technol. Lett., vol. 25, no. 15 , pp. $1524-1526,2013$.

[30] H. Chen et al., "100Gb/s PolMux-NRZ-AOS-OFDM transmission system," Opt. Express, vol. 17, no. 21, pp. 18768-18773, 2009.

[31] A. Dochhan et al., "Flexible bandwidth $448 \mathrm{~Gb} / \mathrm{s}$ DMT transmission for next generation data center inter-connects," in Proc. ECOC, Cannes, France, 2014,

[32] Y. Zhang et al., "Theoretical and experimental investigation of compatible SSB modulation for single channel long-distance optical OFDM transmission," Opt. Express, vol. 18, no. 16, pp. 16751-16764, 2010.

[33] S.A. Nezamalhosseini et al., "Theoretical and experimental investigation of direct detection optical OFDM transmission using beat interference cancellation receiver," Opt. Express, vol. 21, no. 13, pp. 15237-15246, 2013.

[34] W.-R. Peng et al., "Spectrally efficient direct-detected OFDM transmission incorporating a tunable frequency gap and an iterative detection techniques," J. Lightw. Technol., vol. 27, no. 24, pp. 5723-5735, 2009.

[35] Z. Cao et al., "Direct-detection optical OFDM transmission system without frequency guard band," IEEE Photon. Technol. Lett., vol. 22, no. 11, pp. 736-738, 2010.

[36] T. Jiang and Y. Wu, "An overview: Peak-to-average power ratio reduction techniques for OFDM signals," IEEE Trans. on Broadcast., vol. 54, no. 2, pp. 257-268, 2008

[37] J. Armstrong, "OFDM for optical communications," J. Lightw. Technol., vol. 27 , no. 3 , pp. $189-204,2009$.

[38] C.R. Berger et al., "Theoretical and experimental evaluation of clipping and quantization noise for optical OFDM," Opt. Express, vol. 19, no. 18, pp. 17713-17728, 2011.

[39] M.S. Erkılınç et al., "Performance comparison of single sideband direct detection Nyquist-subcarrier modulation and OFDM," to be published in J. Lightw. Technol., vol. 33, no. 10, pp. 2038-2046, 2015.

[40] A.O.J. Wiberg et al., "Single cycle subcarrier modulation," in Proc. OFC, San Diego, CA, USA, 2009, paper OTuE.1.

[41] A.S. Karar and J.C. Cartledge, "Generation and detection of a $112-\mathrm{Gb} / \mathrm{s}$ dual polarization signal using a directly modulated laser and half-cycle 16-QAM Nyquist-subcarrier-modulation," in Proc. ECOC, Amsterdam, Netherlands, 2012, post-deadline paper Th3.A.4.

[42] G. Bosco et al., "Performance limits of Nyquist-WDM and CO-OFDM in high-speed PM-QPSK systems," IEEE Photon. Technol. Lett., vol. 22, no. 15, pp. 1129-1131, 2010.

[43] M.S. Erkı1lınç et al., "Nyquist-shaped dispersion-precompensated subcarrier modulation with direct detection for spectrally-efficient WDM transmission," Opt. Express, vol. 22, no. 8, pp. 9420-9431, 2014.

[44] M.S. Erkilinc et al., "Spectrally-efficient single-sideband subcarriermultiplexed quasi-Nyquist QPSK with direct detection," in Proc. ECOC, London, UK, 2013, paper Tu3C4.

[45] R.I. Killey et al., "Electronic dispersion compensation by signal predistortion using digital processing and a dual-drive Mach-Zehnder modulator," IEEE Photon. Technol. Lett., vol. 17, no. 3, pp. 714-716, 2005.

[46] J. McNicol et al., "Electrical domain compensation of optical dispersion [optical fibre communication applications]," in Proc. OFC, Anaheim, CA, USA, 2005, paper OThJ3.

[47] G.P. Agrawal, "Applications of nonlinear fiber optics," 3rd ed., Academic press, 2010.

[48] C.E. Shannon, "A mathematical theory of communication," Bell Syst. Technol. J., vol. 27, no. 3, pp. 379-423, 1948. 
[49] N. Liu, C. Ju and X. Chen, "Nonlinear ISI cancellation in VSSB Nyquist-SCM system with symbol pre-distortion," Opt. Commun., vol. 338 , pp. 492-495, 2015.

M. Sezer Erkılınç received his B.Sc. and M.Sc. degree in Electrical \& Electronic Engineering from Koç University, Istanbul, Turkey in 2009 and Rochester Institute of Technology, Rochester, NY, USA in 2011, respectively. $\mathrm{He}$ is pursuing a Ph.D. degree in the Optical Networks Group in the Department of Electronic \& Electrical Engineering at the University College London in London, UK since November 2011. He is currently investigating spectrallyefficient modulation formats in direct detection links and simplified coherent receiver architectures for access and metropolitan/regional applications. He is a graduate student member of IEEE and SPIE.

Zhe Li Zhe Li received his B.Eng. degree in optical information science and technology from Jilin University, China, in 2011. The following September, he joined University College London (UCL) to pursue an MSc in Wireless and Optical Communications. In December 2013, Zhe joined the Optical Networks Group at UCL as an MPhil/PhD candidate. Under the supervision of Dr. Robert Killey, he is currently investigating spectrally-efficient and low-cost optical communication systems with direct detection. He is a graduate student member of IEEE.

Stephan Pachnicke (M'09-SM'12) received the M.Sc. degree in Information Engineering from City University, London, U.K. in 2001 and the Dipl.-Ing. and Dr.-Ing. degrees in electrical engineering from TU Dortmund, Germany, in 2002 and 2005, respectively. In 2005 he also got the Dipl.-Wirt.-Ing. degree in business administration from Fern Universitt, Hagen, Germany. In January 2012, he finished his habilitation on optical transmission networks and since then also works as Privatdozent (adjunct professor) at TU Dortmund. From 2007 until 2011 he was working as Oberingenieur at the Chair for High Frequency Technology, TU Dortmund. Currently he is with ADVA Optical Networking SE in the Advanced Technology Group (CTO Office), where he is leading EU-funded research projects on next generation optical access and fixed-mobile convergence. He is author or co-author of more than 80 scientific publications as well as author of a book on Fiber-Optic Transmission Networks (Springer, 2011). He is a member of VDE/ITG and a senior member of IEEE.

Helmut Griesser is a Principal Engineer in the Advanced Technology group at ADVA Optical Networking, Munich, Germany. Current research interests include signal processing, coding, modulation formats and system design for optical fiber communication systems. From 2002 till 2011, he worked with Marconi and Ericsson on high-speed fiber transmission. He holds a Dr.-Ing. and Dipl.-Ing. degree in electrical engineering from the University of Ulm, Germany.
Benn C. Thomsen (M'06) received the B.Tech. degree in optoelectronics and M.Sc. and Ph.D. degrees in physics from The University of Auckland, Auckland, New Zealand. His doctoral research involved the development and characterization of short optical pulse sources suitable for high-capacity optical communication systems. He then joined the Optoelectronics Research Centre, Southampton University, U.K., as a Research Fellow in 2002, where he carried out research on ultra short optical pulse generation and characterization, optical packet switching based on optically coded labels, and all-optical pulse processing. He joined the Optical Networks Group, University College London, London, U.K., in 2004, and held an EPSRC Advanced Fellowship from 2006 to 2011 and was appointed as a lecturer in 2007. He is currently a senior lecturer at UCL and his research focuses on optical transmission, physical-layer implementation of dynamic optical networking technology and the development of high capacity multimode fibre systems exploiting MIMO DSP.

Polina Bayvel received her B.Sc. (Eng) and Ph.D. degrees in Electronic \& Electrical Engineering from University of London, UK, in 1986 and 1990, respectively. In 1990, she was with the Fiber Optics Laboratory, General Physics Institute, Moscow (Russian Academy of Sciences), under the Royal Society Postdoctoral Exchange Fellowship. She was a Principal Systems Engineer with STC Submarine Systems, Ltd., London, UK, and Nortel Networks (Harlow, UK, and Ottawa, ON, Canada), where she was involved in the design and planning of optical fibre transmission networks. During 1994-2004, she held a Royal Society University Research Fellowship at University College London (UCL), and in 2002, she became a Chair in Optical Communications and Networks. She is currently the Head of the Optical Networks Group, UCL. She has authored or co-authored more than 350 refereed journal and conference papers. Her research interests include wavelength-routed optical networks, high-speed optical transmission, and the mitigation of fibre nonlinearities.

Prof. Bayvel is a Fellow of the Royal Academy of Engineering (FREng.), the Optical Society of America, the UK Institute of Physics, and the Institute of Engineering and Technology. She was the recipient of the Royal Society Wolfson Research Merit Award (2007-2012), 2013 IEEE Photonics Society Engineering Achievement Award and 2014 Royal Society Clifford Patterson Prize Lecture and Medal.

Robert I. Killey (M'00) received the B.Eng. degree in electronic and communications engineering from the University of Bristol, U.K., in 1992 and the M.Sc. degree from University College London (UCL) in 1994. He received the D.Phil. degree from the University of Oxford, U.K., in 1998. Following this, he joined the Optical Networks Group at UCL where is currently Reader in Optical Communications. He serves on the technical program committees of ECOC and OFC conferences, was a member of the conference committees of ACP, OECC and IEEE LEOS Annual Meetings and was General Cochair of the Signal Processing for Photonic Communications (SPPCom) meeting at the OSA Advanced Photonics Congress. He was an Associate Editor of the IEEE/OSA Journal of Optical Communications and Networking. His research interests include nonlinear fiber effects in WDM transmission systems, advanced modulation formats and digital signal processing for optical communications. 